\title{
2 Correction of basic equations for deep bed filtration with dispersion
} \\ J.E. Altoé F. ${ }^{\text {a }}$, P. Bedrikovetsky ${ }^{\text {a,* }}$, A.G. Siqueira ${ }^{\text {b }}$, A.L.S. de Souza ${ }^{\text {b }}$, F.S. Shecaira ${ }^{\text {b }}$ \\ ${ }^{a}$ Department of Petroleum Exploration and Production Engineering, North Fluminense State University Lenep/UENF, \\ Rod. Amaral Peixoto, km 163 - Av. Brenand, s/n Imboacica - Macaé, RJ 27.925-310, Brazil \\ ${ }^{\mathrm{b}}$ Petrobras/Cenpes, Cidade Universitaria Q.7, Ilha Do Fundão, 21949-900 - Rio De Janeiro - RJ, Brazil
}

Accepted 4 November 2005

Abstract

Deep bed filtration of particle suspensions in porous media occurs during water injection into oil reservoirs, drilling fluid invasion into reservoir productive zones, fines migration in oil fields, bacteria, virus or contaminant transport in groundwater, industrial filtering, etc. The basic features of the process are advective and dispersive particle transport and particle capture by the porous medium.

Particle transport in porous media is determined by advective flow of carrier water and by hydrodynamic dispersion in microheterogeneous media. Thus, the particle flux is the sum of advective and dispersive fluxes. Transport of particles in porous media is described by an advection-diffusion equation and by a kinetic equation of particle capture. Conventional models for deep bed filtration take into account hydrodynamic particle dispersion in the mass balance equation but do not consider the effect of dispersive flux on retention kinetics.

In the present study, a model for deep bed filtration with particle size exclusion taking into account particle hydrodynamic dispersion in both mass balance and retention kinetics equations is proposed. Analytical solutions are obtained for flows in infinite and semi-infinite reservoirs and in finite porous columns. The physical interpretation of the steady-state flow regimes described by the proposed and the traditional models favours the former.

Comparative matching of experimental data on particle transport in porous columns by the two models is performed for two sets of laboratory data.

(C) 2005 Published by Elsevier B.V.

Keywords: Deep bed filtration; Dispersion; Suspension; Governing equations; Modelling; Porous media; Emulsion

\section{Introduction}

Severe injectivity decline during sea/produced water injection is a serious problem in offshore waterflood projects. The permeability impairment occurs due to capture of particles from injected water by the rock.

\footnotetext{
* Corresponding author. Tel.: +55 22 27733391; fax: +55 22 27736565 .

E-mail addresses: pavel@lenep.uenf.br, pavel.russia@globo.com (P. Bedrikovetsky).
}

The reliable modelling-based prediction of injectivity decline is important for the injected-water-treatment design, for injected water management (injection of sea- or produced water, their combinations, water filtering), etc.

The formation damage induced by penetration of drilling fluid into a reservoir also occurs due to particle capture by rocks and consequent permeability reduction. Other petroleum applications include sand production control, fines migration and deep bed filtration in gravel packs. 
The basic equations for deep bed filtration taking into account advective particle transport and the kinetics of particle retention, and neglecting hydrodynamic dispersion have been derived essentially following the filtration equation proposed by Iwasaki (1937). A number of predictive models have been presented in the literature (Sharma and Yortsos, 1987a,b,c; Elimelech et al., 1995; Tiab and Donaldson, 1996, Khilar and Fogler, 1998; Logan, 2001). The equations allow for various analytical solutions, which have been used for the treatment of laboratory data and for prediction of porous media contamination and clogging (Herzig et al., 1970; Pang and Sharma, 1994; Wennberg and Sharma, 1997; Bedrikovetsky et al., 2001, 2002).

However, particle dispersion in heterogeneous porous media is important for both small and large scales (Lake, 1989; Jensen et al., 1997). The typical core sizes in laboratory experiments are small, and hence the Peclet number is relatively high. The typical dispersivity values for large formation scales are high, and consequently the Peclet number may also take high values. The Peclet number for either situation may amount up to 10-20.

The effect of dispersion on deep bed filtration is particularly important near to wells, where the dispersivity may already arise to the bed scale, and the formation damage occurs in one two-meter neighbourhood.

Therefore, several deep bed filtration studies take into account dispersion of particles (Grolimund et al., 1998; Kretzschmar et al., 1997; Bolster et al., 1998; Unice and Logan, 2000; Logan, 2001; Tufenkji et al., 2003). A detailed description of such early work is presented in the review paper by Herzig et al. (1970). The models developed account for particle dispersion in the mass balance for particles but do not consider the dispersion flux contribution to the retention kinetics.

In the present study, the proposed deep bed filtration model takes into account dispersion in both the equation of mass balance and in that of capture kinetics. Several analytical models for constant filtration coefficient and for dynamic blocking filtration coefficient have been developed. If compared with the traditional model, the proposed model exhibits more realistic physics behaviour. The difference between the traditional and proposed model is significant for small Peclet numbers.

The structure of the paper is as follows. In Section 2 we formulate the corrected model for deep bed filtration of particulate suspensions in porous media accounting for hydrodynamic dispersion of suspended particles. The dispersion-free deep bed filtration model is presented in Section 3 as a particulate case of the general system with dispersion. The analytical models for flow in infinite and semi-infinite reservoirs for constant filtration coefficient are presented in Sections 4 and 5 , respectively. An analytical solution for deep bed filtration in semi-infinite reservoirs with the fixed inlet concentration is given in Section 6. Analytical steady state solution for laboratory coreflood in discussed in Section 7. The analytical models allow for laboratory data treatment (Section 8). Travelling wave flow regimes for dynamic blocking filtration coefficient are described in Section 9. In Section 10, three dimensional equations for deep bed filtration with dispersion are derived. Mathematical details of the derivations are presented in Appendices. Dimensionless form of governing equations and initial-boundary conditions are given in Appendix A. The transient solutions for flow in infinite and semi-infinite reservoirs and constant filtration coefficient are derived in Appendices B, C and D. Appendix E contains derivations for steady state solution in a finite core. Appendix F contains derivations for travelling wave flow.

\section{Model formulation}

Let us derive governing equations for deep bed filtration taking into account particle dispersion. The usual assumptions of constant suspension density and porosity for low particle concentrations are adopted. The balance equation for suspended and retained particles (Iwasaki, 1937; Herzig et al., 1970) is:

$\frac{\partial}{\partial t}(\phi c+\sigma)+\frac{\partial q}{\partial x}=0$

Here, the concentration $c$ is a number of suspended particles per unit volume of the fluid, and the retained particle concentration $\sigma$ is a number of captured particles per unit volume of the rock.

The particle flux $q$ consists of the advective and dispersive components:

$q=U c-D \frac{\partial c}{\partial x}$

$D=\alpha_{D} U$

Here the dispersion coefficient $D$ is assumed to be proportional to the flow velocity $U$, and the proportionality coefficient $\alpha_{D}$ is called the longitudinal dispersivity (Lake, 1989; Nikolaevskij, 1990; Sorbie, 1991).

Let us consider the following physical model for the size exclusion particle capture in porous media (Santos and Bedrikovetsky, 2005). Particles are not captured during flow through the pore system, but there is a 
143 sequence of particle capturing sieves perpendicular to 144 the flow direction. The probability for a particle to be 145 captured is equal to $\lambda l$ ( $l$ is the distance between the 146 sieves), and that to pass through is $1-\lambda l$. In other 147 words, after particles pass the distance $l$, their flux 148 reduces $1-\lambda l$ times.

149 So, the so called filtration coefficient $\lambda$ is determined 150 through the fraction $\lambda l$ of the particle flux that remains 151 in porous media during flow along the distance $l$ (Iwa152 saki, 1937). The filtration coefficient $\lambda$ is the probabil153 ity for particle to be captured during the flow over the 154 unit distance; its dimension is $L^{-1}$.

155 Following the probabilistic interpretation of filtra156 tion coefficient, Herzig et al. (1970) have calculated 157 the number of captured particles per unit time per unit 158 volume during advective flow. Let us calculate the 159 deposition rate for advective-dispersive flow. The 160 number of particles crossing sieve during time $\Delta t$ is 161 equal to $q A \Delta t$, where $A$ is a cross section area. The 162 particles move along the distance $U \Delta t / \varphi$ during this 163 time, here $\varphi$ is the porosity. The probability for 164 particle to be captured is $\lambda U \Delta t / \varphi$. Particle retention 165 takes place in the volume $A U \Delta t / \varphi$. The deposition 166 rate is

$$
\frac{\Delta \sigma}{\Delta t}=\frac{(q A \Delta t)(\lambda U \Delta t / \phi)}{(A U \Delta t / \phi) \Delta t}=\lambda q
$$

168 So, the interpretation of the capture rate in terms of 170 probability for the particle capture in elementary refer171 ence volume implies that the capture rate is proportion172 al to the total particle flux (Eq. (2)) rather than just to its 173 advective component.

174 From now on we assume that the particle capture 175 rate is proportional to the overall particle flux (first176 order particle retention kinetics):

$$
\frac{\partial \sigma}{\partial t}=\lambda(\sigma) q
$$

178 Here the filtration coefficient $\lambda(\sigma)$ is a function of 180 retained concentration $\sigma$. Particle deposition changes 181 the pore space geometry and, consequently, the condi182 tions for size exclusion capture, so the deposition rate 183 should be retained-concentration-dependent.

184 Fig. 1 illustrates size exclusion capture of particles 185 - a pore captures a particle if the particle size exceeds 186 the pore size, otherwise the particle passes through the 187 pore. Therefore, the capture rate must be proportional to 188 the total particle flux. A particle is captured by a pore 189 regardless of whether the advective or dispersive flux 190 has brought the particle to the pore.

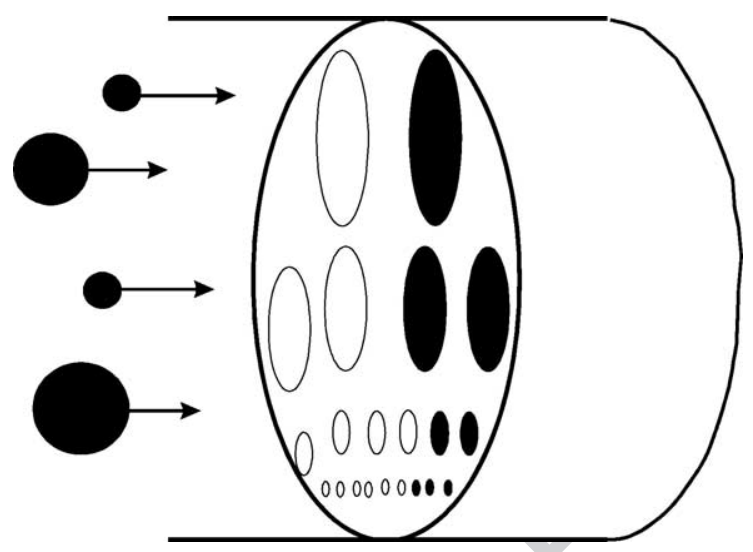

Fig. 1. Schema for particle capture by size exclusion in deep bed filtration.

The same applies to bridging build-up and to the consequent particle capture (Payatakes et al., 1974; Elimelech et al., 1995).

It is worth mentioning that usually size exclusion is not dominant in virus and bacteria capture during their flow in porous media. The retention mainly happens due to sorption (Kuhnen et al., 2000). In this case, the authors assume that the deposition rate is proportional to suspended concentration only. The proportionality coefficient dimension is $1 / T$, i.e. the proportionality coefficient is a probability for particle to be captured during the unitary time. In this case, neither hydrodynamic dispersion nor advective velocity enters in the capture rate expression.

The same applies to chemical reactions and dissolution in porous media (Kechagia et al., 2002).

Many experiments show that during the particle suspension flow in porous media, the particle capture rate rapidly decreases as particles start to accumulate on the collectors; the retention stops when the retained concentration reaches some critical value (Elimelech et al., 1995; Kuhnen et al., 2000). This phenomenon is called blocking. It can be explained by decrease of the number of vacancies for further retention during the retention process.

For example, if the injected particle sizes are comparable with pore throats sizes, the particles are captured by the pore size exclusion. Consider a wide throat size distribution, and injection of particles with intermediate sizes. Particles are captured in smaller pores. When all small pores are filled, the suspension flows through thick throats, and the particles are not captured any more.

Hereafter the following features of the filtration blocking coefficient $\lambda(\sigma)$ are assumed: 
226

227 filtration coefficient

$\lambda(\sigma)=\lambda_{0}(1-b \sigma)$

228 so called Langmuir blocking function (Kuhnen et al., 230 2000). It is typical where the capture is realized by a

231 mono-layer adsorption.

232 This case corresponds to the situation where one

233 vacancy can be filled by one particle. So, retention of

234 some particles results in filling of the same number of

235 vacancies, i.e. the total of deposited particle concentra-

236 tion $\sigma(x, t)$ and the vacant pore concentration $h(x, t)$ is

237 equal to initial concentration of vacancies $h(x, 0)$ :

$h(x, t)=h(x, 0)-\sigma(x, t)$

230 The capture rate is proportional to the product of 241 particle flux and vacancy concentration (acting mass 242 law). Using Eq. (7), we obtain:

$\frac{\partial \sigma}{\partial t}=\lambda_{0}\left(1-\frac{\sigma}{h(x, 0)}\right) q$

243 The filtration function $\lambda(\sigma)$ depends on porous media 246 structure. Therefore, for heterogeneous porous media 247 where initial vacancy concentration depends on $x$, the 248 filtration function is $x$-dependent: $\lambda=\lambda(\sigma, x)$. Further in

249 the paper we assume a uniform initial vacancy concen-

250 tration and use the dependency $\lambda=\lambda(\sigma)$.

251 So, the Langmuir linear blocking function (Eq. (6)) 252 corresponds to "one particle - one pore" kinetics [Eq.

253 (8)]. The comparison of formulae Eqs. (8) and (4) 254 results in Eq. (6).

255 If $\lambda_{0}$ in Eq. (8) is also a function of $\sigma$, the blocking 256 filtration coefficient $\lambda(\sigma)$ is non-linear.

257 Darcy's law for suspension flow in porous media 258 includes the effect of permeability decline during par259 ticle retention:

$$
U=-\frac{k_{0} k(\sigma)}{\mu} \frac{\partial p}{\partial x}
$$

260

$k(\sigma)=\frac{1}{1+\beta \sigma}$

$26 B$ Here $k(\sigma)$ is called the permeability reduction function,

264 and $\beta$ is the formation damage coefficient.

265 Eqs. (1), (2), (4) and (9) form a closed system of four 266 equations that govern the colloid filtration with size

267 exclusion particle capture in porous media. The 268 unknowns are suspended concentration $c$, deposited $269 \sigma$, particle flux $q$ and pressure $p$.

270 The independence of the filtration and dispersion 271 coefficients of pressure allows separation of Eqs. (1), 272 (2) and (4) from Eq. (9), which means that the sus273 pended and retained concentrations and the particle flux can be found from the system of Eqs. (1), (2) and (4) and then the pressure distribution can be found from Eq. (9).

Form of the system of governing equations in non-dimensional co-ordinates is presented in Appendix A, (Eqs. (A-2)-(A-5)). The system contains the dimensionless parameter $\varepsilon_{D}$ that is the inverse to the Peclet number; it is equal to the dispersion-to-advective flux ratio (Nikolaevskij, 1990). From Eq. (3) it follows that:

$\varepsilon_{D}=\frac{1}{\mathrm{Pe}}=\frac{D}{L U}=\frac{\alpha_{D}}{L}$

The dispersion-advective ratio $\varepsilon_{D}$ is equal to the ratio between the micro heterogeneity size $\alpha_{D}$ (dispersivity) and the reference size of the boundary problem $L$.

Let us estimate the contribution of dispersion to the total particle flux (Eq. (2)). In the majority of papers, deep bed filtration model has been modelled under the laboratory floods conditions, where homogeneous sand columns are employed (Elimelech et al., 1995; Unice and Logan, 2000; Tufenkji et al., 2003). On the core scale in homogeneous cores, we have $L \sim 0.1 \mathrm{~m}$, $\alpha_{D} \sim 0.001 \mathrm{~m}, \varepsilon_{D} \sim 0.01$, and hence the dispersion can be neglected. In natural heterogeneous cores, $\varepsilon_{D}$ can amount to 0.1 or more, and dispersion should be taken into account (Lake, 1989; Bedrikovetsky, 1993). In a well neighbourhood, the reference radius of formation damage zone is $1 \mathrm{~m}$, the heterogeneity reference size is also $1 \mathrm{~m}$, so $\varepsilon_{D}$ has order of magnitude of unity.

The dispersivity $\alpha_{D}$ can reach several tens or even hundreds of meters at formation scales (Lake, 1989; Jensen et al., 1997); thus, the dimensionless dispersion can have the order of magnitude of unity, and hence hydrodynamic dispersion should be taken into account.

Now we formulate one dimensional problem for suspension injection into a porous core/reservoir.

The absence of suspended and retained particles in porous media before the injection is represented by the initial conditions:

$t=0: c=\sigma=0$

Fixing the inlet particle flux during the injection of particulate suspension in a reservoir determines the boundary condition:

$x=0: U c-D \frac{\partial c}{\partial x}=c^{0} U$

Sometimes the dispersive term in the boundary condition (Eq. (13)) is neglected (van Genuchten, 1981 and Nikolaevskij, 1990):

$x=0: c=c^{0}$ 
325 The particle motion in porous media can be decom326 posed into an advective flow with constant velocity and 327 the dispersive random walks around the front that 328 moves with advective velocity (Kampen, 1984). It is 329 assumed that once a particle leaves the core outlet by 330 advection it cannot come back by dispersion. This 331 assumption leads to the boundary condition of absence 332 of dispersion at the core outlet (Danckwerts, 1953; 333 Nikolaevskij, 1990):

$x=L: \frac{\partial c}{\partial x}=0$

336 In dimensionless coordinates (Eq. (A-1)), the pro337 posed model with a constant filtration coefficient takes 338 the form (Eqs. (A-10) and (11)):

$$
\frac{\partial C}{\partial T}+v \frac{\partial C}{\partial X}=\varepsilon_{D} \frac{\partial^{2} C}{\partial X^{2}}-\Lambda C
$$

$339 v=1-\Lambda \varepsilon_{D}$

340 Neglecting the dispersion term in the capture kinet343 ics Eqs. (16) and (17) results in:

$$
\frac{\partial C}{\partial T}+\frac{\partial C}{\partial X}=\varepsilon_{D} \frac{\partial^{2} C}{\partial X^{2}}-\Lambda C
$$

346 Eq. (18) is a traditional advective-diffusive model 347 with a sink term. The boundary condition (Eq. (13)) 348 fixes the inlet flux in this model.

349 Eq. (16) looks like the advective-diffusive model 350 (Eq. (18)) with advective velocity $v$, and seems this 351 velocity should appear in the expression for the inlet 352 flux (Eq. (13)). However, the real advective velocity in $353 \mathrm{Eq}$. (16) is equal to one, and the delay term $-\Lambda \varepsilon_{D}$ 354 appears due to the capture of particles transported by 355 the dispersive flux and is not a part of the flux. From 356 conservation law (Eqs. (1) and (A-2)) it follows that the 357 particle flux is continuous at the inlet; the boundary 358 condition for Eq. (16) should be given by Eq. (13) that 359 differs from the inlet boundary condition for the equiv360 alent advective-diffusive model with the advective 361 velocity $v$.

362 Following Logan (2001), from now on the model 363 (Eq. (18)) will be referred to as the HLL model in order 364 to honour the fundamental work by Herzig et al. 365 (1970).

366 The difference between the presented and the HLL 367 model is the delay term $\Lambda \varepsilon_{D}$ that appears in the advec368 tive flux velocity (Eq. (17)). This is the collective effect 369 of the particle dispersion and capture. Appearance of 370 the delay term $\Lambda \varepsilon_{D}$ in the advective flux velocity (Eq. 371 (17)) is due to accounting for diffusive flux in the 372 capture kinetics (Eq. (2) and Eq. (4)).
A delay in the particle pulse arrival to the column effluent if compared with the tracer pulse breakthrough was observed by Massei et al. (2002).

The length $L$ used in dimensionless parameters (Eq. (A-1)) is a reference size of the boundary problem. It affects the dimensionless filtration coefficient $\Lambda$ (Eq. (A-1)) and the inverse to Peclet number $\varepsilon_{D}$, (Eq. (11)) and drops out the delay term: $\Lambda \varepsilon_{D}=\lambda \alpha_{D}$. The dimensionless time $T$ corresponding to the length $L$ (Eq. (A$1)$ ) is measured in "pore volume injected", which is the common unit in laboratory coreflooding and in field data presentation.

Also, often the injected suspension is traced, and the particle breakthrough curves are presented together with tracer curves (Jin et al., 1997; Ginn, 2000). In this case, the inverse to Peclet number $\varepsilon_{D}$ in Eq. (16) is already known from the tracer data and it is convenient to use dimensionless variables and parameters (Eq. (A-1)).

Nevertheless, Eq. (16) depends on two independent dimensionless parameters $-\varepsilon_{D}$ and $\Lambda$.

The inverse to filtration coefficient is an average penetration depth of suspended particles (Herzig et al., 1970), so the inverse to reference value of filtration coefficient $1 / \lambda_{0}$ can be used as a reference length in dimensionless linear co-ordinate (Eq. (A-12)). For corresponding dimensionless variables (Eq. (A-12)), the dimensionless $\Lambda$ becomes equal to unit in the case of constant filtration coefficient, and Eq. (16) becomes dependent of one dimensionless parameter $\varepsilon$ only, (Eq. (A-13)):

$$
\begin{aligned}
& \frac{\partial C}{\partial T^{\prime}}+v \frac{\partial C}{\partial X^{\prime}}=\varepsilon \frac{\partial^{2} C}{\partial X^{\prime 2}}-C \\
& v=1-\varepsilon, \varepsilon=\lambda_{0} \alpha_{D}
\end{aligned}
$$

In the case of filtration function $\Lambda=\Lambda(S)$, Eq. (16) includes deposited concentration $S$, and the model consists of two equations

$$
\begin{aligned}
& \frac{\partial C}{\partial T}+\left(1-\Lambda(S) \varepsilon_{D}\right) \frac{\partial C}{\partial X}=\varepsilon_{D} \frac{\partial^{2} C}{\partial X^{2}}-\Lambda(S) C \\
& \frac{\partial S}{\partial T}=\Lambda(S)\left(1-\varepsilon_{D} \frac{\partial C}{\partial X}\right)
\end{aligned}
$$

for dimensionless parameters (Eq. (A-1)).

For dimensionless variables (Eq. (A-12)), $\varepsilon_{D}$ must be changed to $\varepsilon=\lambda_{0} \alpha_{D}$.

The HLL in this case is also obtained by neglecting dispersion term in capture rate expression.
373

374

375

376

377

378

379

380

381

382

383

384

385

386

387

388

389

390

391

392

393

394

395

396

397

398

399

400

401

402

403

404

$40 \overline{6}$

408

409

412

413

414

415 
416 The order of the governing system (Eq. (20)) can be 417 reduced by one. Introducing the function

$\Phi(S)=\int_{0}^{S} \frac{1}{\Lambda(s)} d s$

418 from Eq. (A-3) we obtain:

$\frac{\partial \Phi(S)}{\partial T}=Q$

420 Substitution of Eq. (22) into Eq. (A-2) results in $\frac{\partial}{\partial T}(C+S)+\frac{\partial}{\partial X}\left(\frac{\partial \Phi(S)}{\partial T}\right)=0$

423 Changing order of differentiation in the second term in 426 the right hand side of Eq. (23) and integrating in $T$ from 427 zero to $T$, we obtain first order partial differential 428 equation:

$C+S+\frac{\partial \Phi(S)}{\partial X}=0$

430 The integration constant that should appear in right 432 hand side of Eq. (24) was calculated from initial con433 ditions (Eq. (12)) - it is equal zero.

434 Eq. (22) becomes

$\frac{\partial \Phi(S)}{\partial T}=1-\varepsilon_{D} \frac{\partial C}{\partial X}$

436 Eqs. (24) and (25) form quasi-linear system of first 438 order equations modeling deep bed filtration with size 439 exclusion particle capture accounting for dispersion.

\section{3. Dispersion free model}

441 Neglecting the dispersion in Eq. (16) results in the 442 simplified deep bed filtration model (Sharma and Yort443 sos, 1987a,b,c; Elimelech et al., 1995; Tiab and 444 Donaldson, 1996):

$\frac{\partial C}{\partial T}+\frac{\partial C}{\partial X}=-\Lambda C$

446 The boundary condition (Eq. (13)) automatically takes 448 the form of Eq. (14).

449 The solution of the dispersion-free deep bed filtra450 tion problem (Eqs. (26) (12) and (14)) is given by

$C(X, T)= \begin{cases}\exp (-\Lambda X) & X \leq T \\ 0 & X>T\end{cases}$

45B Concentration is zero ahead of the concentration front $454 X_{0}(T)=T$. Particles arrive at the column outlet after one 455 pore volume injection. Once the advancing front passes 456 a given location, a steady concentration distribution is 457 immediately established behind the front.

\section{Transient flow in infinite reservoir}

458

Let us consider flow in an infinite reservoir where, initially, water with particles fills the semi-infinite reservoir $X<0$, and clean water fills the semi-infinite reservoir $X>0$. Formula for concentration wave propagation (Eq. (B-2)) is presented in Appendix B.

Fig. 2 shows the concentration profiles for the times $T=0.1,1.0$ and 4.0 with $\varepsilon_{D}=1.0$ and $\Lambda=0.5$. Solid lines correspond to the proposed model and dotted lines correspond to the HLL model. Both models exhibit advective propagation of the concentration wave with diffusive smoothing of the initial shock; the mixture zone expands with time. Suspended concentration is zero ahead of the mixture zone. Behind the mixture zone, concentration does not vary along the reservoir and exponentially decays with time due to deep bed filtration with a constant filtration coefficient. One can observe a delay in the concentration front propagation for the proposed model (Eq. (16)) if compared with the HLL model (Eq. (18)). The difference in the profiles in the two models appears in the mixture zone, while the concentrations ahead of and behind the mixture zone coincide for both models.

5. Analytical model for suspension injection into semi-infinite reservoir

481 482

In this section we consider the particulate suspension injection into a semi-infinite reservoir, $X>0$. The expression for suspension concentration (Eq. (C-2)) is presented in Appendix C.

Fig. 3 epicts particle flux profiles for the dispersi shows the concentration profiles at the moments $T=0.5,1.0,2.0$ and 6.0 as obtained by explicit formula

483 484 485 486 487 488 489

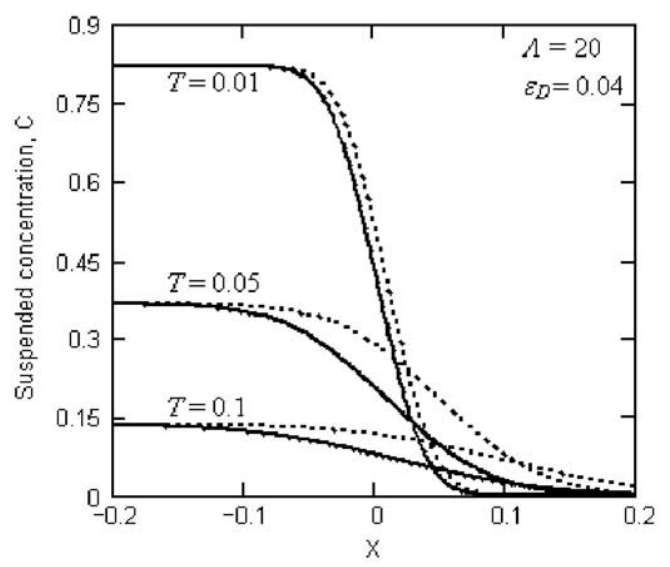

Fig. 2. Concentration wave dynamics in an infinite reservoir by the presented model and by the HLL model. 


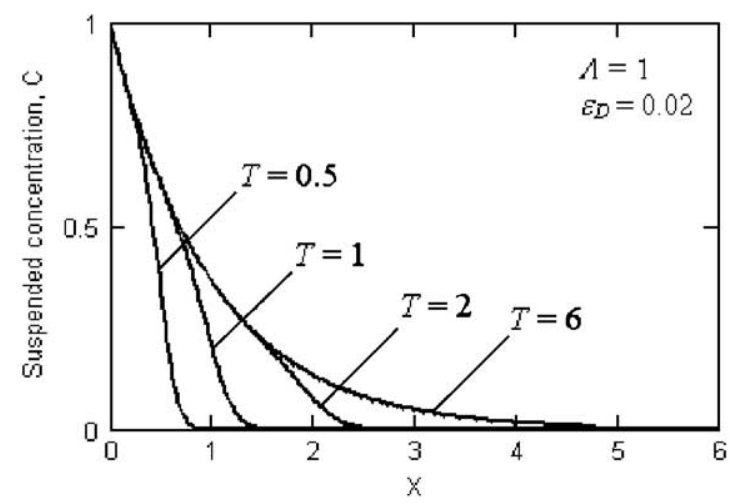

Fig. 3. Dynamics of concentration waves in a semi-infinite reservoir.

490 (Eq. (C-2)). The envelope curve corresponds to the 491 steady-state solution (Eq. (C-5)). Furthermore, for any 492 moment $T$ there exists such position of a mixture zone $493 X_{0}(T)$ that the transient and steady-state profiles behind 494 the zone $\left(X<X_{0}(T)\right)$ almost coincide. Once the transi495 tion zone passes a given location, the steady-state sus496 pended concentration distribution is established behind. 497 After establishing the steady state, all newly arrived 498 particles are captured by the rock, and the suspended 499 concentration is time-independent.

500 The term "steady state" is applied to the suspended 501 concentration only. The retained concentration 502 increases during the flow.

503 The particle flux profile in the steady-state regime 504 (Eq. (C-6)) shows that the $\lambda$ th fraction of the particle 505 flux is captured under the steady-state conditions, and $506(1-\lambda)$ th fraction passes through. The result must be 507 independent of the particle flux partition into the ad508 vective and dispersive parts, i.e. the formula for the 509 steady-state flux profile must not contain the dispersion 510 coefficient (Eq. (C-6)).

511 Fig. 4 depicts particle flux profiles for the disper512 sion-free model (solid line) and for the proposed model 513 using the solution (Eq. (C-5)) (dotted line) for $514 \varepsilon_{D}=0.002$ and $\Lambda=1$. The suspended concentration

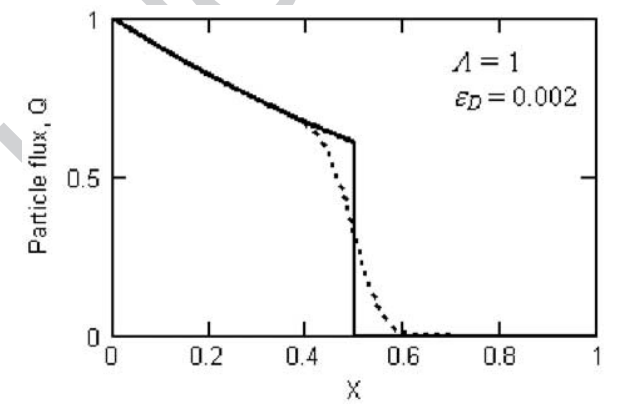

Fig. 4. Particle flux profile for $T=0.5$ (semi-infinite reservoir) by the proposed model and the dispersion-free model. and the particle flux coincide for dispersion-free flow, and the profile is discontinuous. The introduction of particle dispersion leads to smoothing the shock out. The larger is the dispersion coefficient, the wider is the smoothed zone around the shock.

Fig. 5 presents particle flux histories at the point $X=1$ in a semi-infinite reservoir for the dispersion-free case (curve $\varepsilon_{D}=0$ ) and three different dispersion values $\varepsilon_{D}=0.01,0.1$ and 0.5 . On the one side, the higher is the dispersion, the larger is the delay in the arrival of the concentration front. On the other side, the larger is the dispersion, the wider is the mixture zone about the shock. Thus, the effect of the delay in advection competes with that of the dispersion zone expansion. Fig. 5 shows fast breakthrough for large dispersion values.

Let us compare the stationary particle flux profiles behind the moving mixture zone as obtained by the proposed and HLL models. The solution can be obtained from Eq. (C-2) by setting $v=1$ and tending $T$ to infinity. The calculation of the flux profile shows that it is dispersion-dependent.

The asymptotic steady-state particle flux profile (Eq. (C-6)) for the presented model coincides with that for the dispersion-free model and is dispersion-independent.

The comparative results are displayed in Fig. 6. The flux is equal to 0.37 at $X=1$ for both the proposed and dispersion-free models. The HLL model profiles were calculated for $\varepsilon_{D}=0.1,1.0$ and 3.0 ; the corresponding particle fluxes at $X=1$ were found to be $0.40,0.54$ and 0.65 , respectively.

The directions of diffusive and advective fluxes coincide. Therefore, inclusion of the diffusive flux into the particle capture rate (Eq. (4)) increases the retention, and the flux profile as calculated by the proposed model is located below that as obtained by HLL model (Fig. 6).

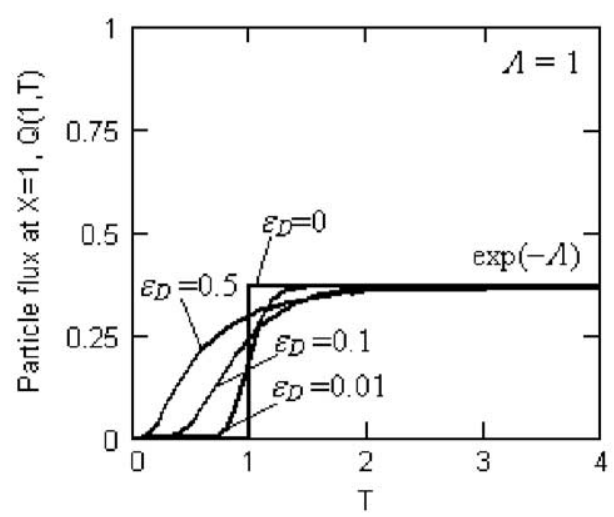

Fig. 5. Particle flux history at the point $X=1$ in semi-infinite reservoir for different dispersion coefficients.
515

516

517

518

519

520

521

522

523

524

525

526

527

528

529

530

531

532

533

534

535

536

537

538

539

540

541

542

543

544

545

546

547

548

549

550 


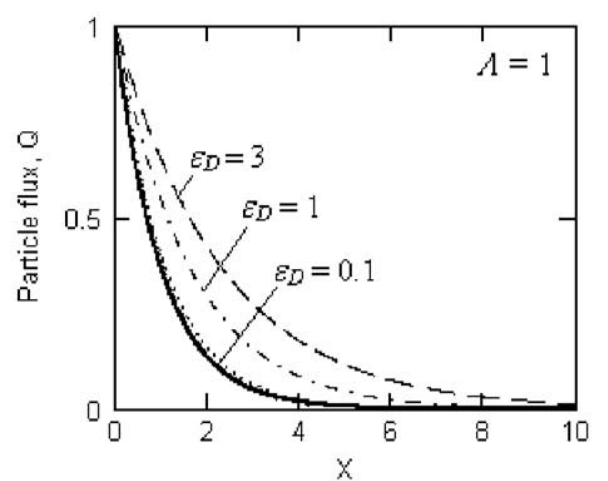

Fig. 6. Steady-state particle flux profiles for the dispersion-free case and by the presented model (the same solid curve) and by the HLL model with $\varepsilon_{D}=0.1, \varepsilon_{D}=1.0$ and $\varepsilon_{D}=3$ (dotted, dot-and-dash and dashed lines, respectively).

551 One may notice a significant difference between the 552 two profiles as calculated by the proposed and the HLL 553 model for large dimensionless dispersion $\left(\varepsilon_{\boldsymbol{D}}=1.0\right.$ and 554 3.0); the difference is negligible for $\varepsilon_{D}$ less than 0.1 . 555 The dependence of the particle flux at $X=1$ on the 556 dimensionless dispersion $\varepsilon_{D}$ is plotted in Fig. 7 for 557 the proposed model (solid line) and the HLL model 558 (dashed line). As mentioned before, the particle flux 559 predicted by the proposed model is independent of 560 dispersion for the steady-state regime (Eq. (C-6)), 561 which implies that the solid line is horizontal.

562 Consider the asymptotic case where the Peclet num563 ber vanishes. As $\varepsilon_{D} \gg 1$, the flux in the HLL model 564 tends to unity; hence the steady-state flux is constant 565 along the column and no particle is captured, which is 566 unphysical. For large dispersion, the advective flux is 567 relatively low; the capture rate in the HLL model is 568 proportional to the advective flux and, therefore, is also

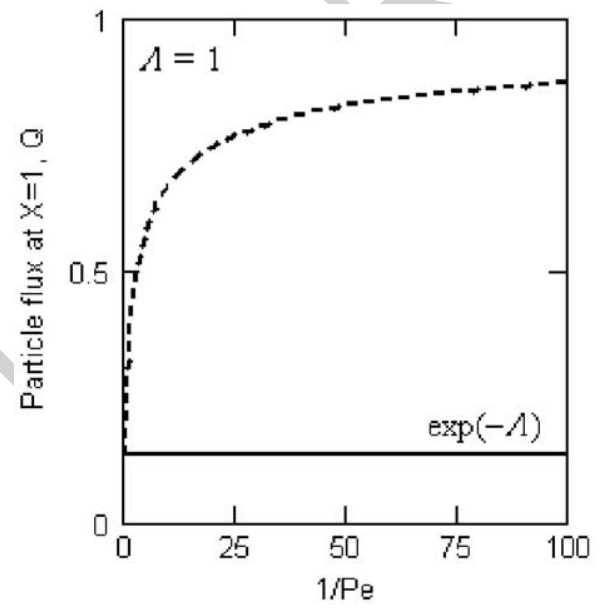

Fig. 7. Effect of dispersion on the flux at $X=1$ for the steady-state mode in a semi-infinite reservoir for proposed and HLL models.

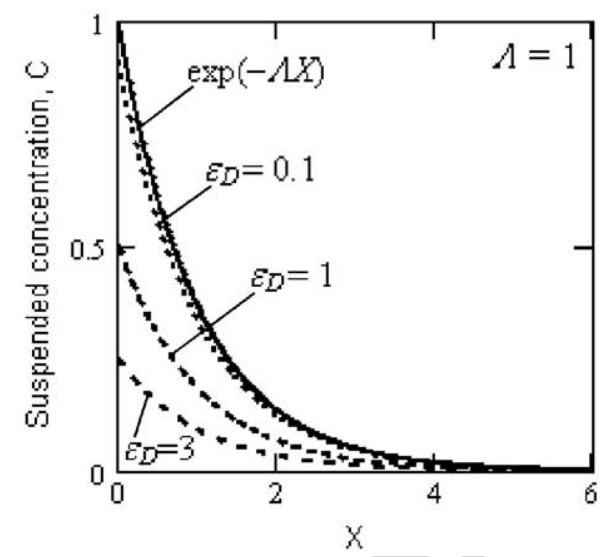

Fig. 8. Comparison between steady-state concentration profiles for deep bed filtration in a semi-infinite reservoir taking into account and neglecting dispersion in the inlet boundary conditions.

low. Thus, particle capture vanishes as $\varepsilon_{D} \gg 1$. No particle retention occurs when the dispersive mass transfer dominates over the advection.

The obtained contradiction occurs because the HLL model does not account for capture of particles transported by dispersive flux (Fig. 8).

\section{Filtration in semi-infinite reservoir with simplified} inlet boundary conditions

Let us discuss the case where dispersion is neglected in the inlet boundary condition (Eq. (14)). The exact solution is obtained in Appendix D, (Eq. (D-1)).

The concentration profile in steady-state regime (Eq. (D-2)) coincides with the concentration profile for the dispersion-free model. The simplified inlet boundary condition (Eq. (14)) is the same as the one for the dispersion-free model. Hence, the introduction of dispersion into the deep bed filtration model while keeping the same boundary condition (Eq. (14)) does not change the asymptotic profile of the suspended concentration.

Fig. 6 shows steady-state concentration profiles for the simplified inlet boundary condition, given by Eq. (D-2) (solid line) and for the complete inlet boundary condition, given by Eq. (C-5) (dotted curves). Three dotted curves correspond to $\varepsilon_{D}=0.1,1.0$ and 3.0. The inlet concentration for dotted lines is always less than unity. The higher is the dispersion the lower is the stationary concentration under the fixed inlet flux.

\section{Steady-state solution for filtration in finite cores}

The expression for the particle flux profile in steadystate regime (Eq. (E-2)) coincides with the flux profile behind the mixture zone in semi-infinite media. The 


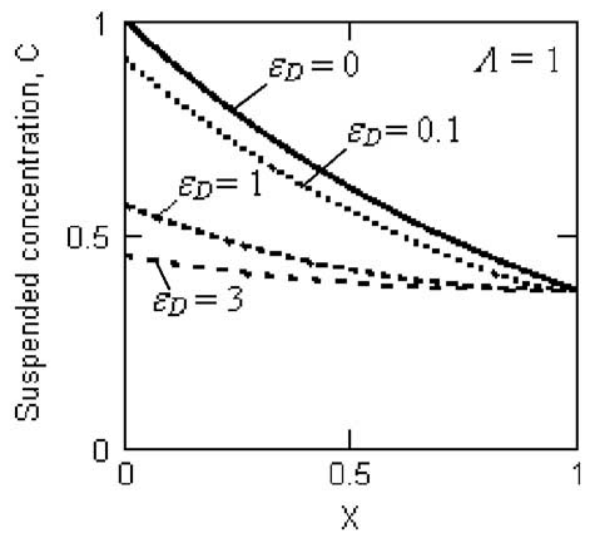

Fig. 9. Steady-state suspended concentration profiles for filtration in a limited core for $\varepsilon_{D}=0,0.1,1.0$ and 3.0 (solid, dotted, dashed and dotand-dash lines, respectively).

600 concentration profiles are different because the bound601 ary condition of the dispersion absence is set at the core 602 outlet $X=1$ for the finite cores and at $X \rightarrow \infty$ for semi603 infinite media.

604 The inlet concentration (Eq. (E-4)) is less than unity. 605 It decreases as dispersion increases. By letting $\varepsilon_{D} \rightarrow \infty$ 606 in Eq. (E-4), we find that the inlet concentration tends 607 to $\exp (-\Lambda)$, which is the outlet concentration (Eq. (E608 5)). Hence, as dispersion tends to infinity, the sus609 pended concentration profile becomes uniform.

610 Fig. 9 shows suspended concentration profiles for $611 \varepsilon_{D}=0.1,1.0$ and 3.0. The inlet concentration for $\varepsilon_{D}=0.1$ 612 is equal to 0.91 . For $\varepsilon_{D}=3.0$ the profile is almost uniform.

613 It is important to emphasize that the outlet concentra614 tion (Eq. (E-5)) is independent of the dispersion coeffi615 cient and is determined by the filtration coefficient only. 616 This fact is in agreement with the presented above 617 interpretation of the filtration coefficient: $\lambda l$ is the prob618 ability for a particle to be captured by the sieve. The 619 outlet concentration coincides with the particle flux due 620 to the outlet boundary condition (Eq. (A-7)). Therefore, 621 the outlet concentration under the steady-state condi622 tions must be determined by the probability for a particle 623 to be captured and must be independent of dispersion. 624 The outlet concentration predicted by the HLL 625 model depends on the dispersion coefficient.

626 It is worth mentioning that the retention profile (Eq. 627 (E-6)) is dispersion independent. This is because the 628 capture rate is proportional to the total particle flux 629 (Eq. (E-2)).

\section{8. Treatment of laboratory data}

631 The formula for steady state limit of the outlet 632 concentration (Eq. (E-5)) allows determining the filtra- tion coefficient from the asymptotic value of the breakthrough curve. From Eq. (E-5) it follows that

$\Lambda=-\ln C(1)$

Formula Eq. (28) coincides with that for determining the filtration coefficient from the asymptotic value of the breakthrough curve using the dispersion-free model (Eq. (26)), see (Pang and Sharma, 1994). The dispersion acts only in the concentration front neighbourhood, the asymptotic value for the breakthrough concentration is dispersion-independent.

Let us find out which model provides better fit to the experimental data. First, we determine the intervals for the test parameters where the difference between the modelling data by the two models is significant.

The proposed and HLL models differ by the delay term $\Lambda \varepsilon_{D}$ in the advective velocity. The models coincide as $\varepsilon_{D}=0$. Hence, the larger is the dispersivity, the higher should be the difference between the two models.

Fig. 10 shows the core outlet flux for the steady-state regime with different $\Lambda$ and $\varepsilon_{D}$ as calculated by the proposed model (solid line) and the HLL model (dashed line). The marked points on dashed curves correspond to the value of $\varepsilon_{D}$ where the difference between the proposed and HLL models starts to exceed $10 \%$. For $\Lambda=4,1$ and 0.55 , the $10 \%$, the difference between the outlet fluxes can be observed for $\varepsilon_{D}$ greater than $0.006,0.13$ and 1.74 , respectively. The value $\Lambda=4$ is typical for seawater injected in medium permeability cores. The value $\Lambda=0.55$ is typical for virus transport in highly permeable porous columns. The typical core size $L=0.1 \mathrm{~m}$. So, in order to validate the proposed and HLL models, one should perform laboratory coreflood with seawater in cores with dispersivity exceeding

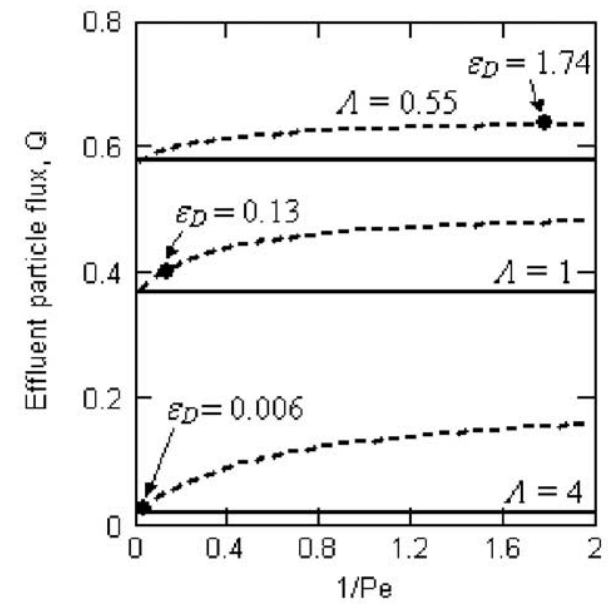

Fig. 10. Effect of dispersion on the particle flux at the core outlet for steady-state flows. 
$68510^{-3} \mathrm{~m}$; the core dispersivity for virus transport should 686 exceed $0.2 \mathrm{~m}$.

687 In papers by Ginn, 2000 and Jin et al., 1997, the 688 outlet concentrations during the injection of particulate 689 suspensions into sand porous columns were measured 690 in laboratory tests. Flow experiments on the transport of 691 oocysts bacteria and pathogenic viruses were carried 692 out in these studies. Laboratory test parameters are 693 presented in Table 1, where the first and the second 694 lines correspond to tests presented in Ginn, 2000, four 695 other tests are taken from the paper by Jin et al., 1997. 696 The breakthrough curves in Fig. 11a, b correspond to 697 tests 2 and 3 . The filtration coefficients are calculated 698 from the asymptotic values $C(X=1, T \rightarrow \infty)$ by Eq. 699 (28) and are presented in Table 2.

700 In the laboratory tests in both works, the injected 701 water was traced, and the tracer outlet concentrations 702 were measured. Chloride and bromide tracers were used 703 in order to determine the dispersion coefficient. The 704 particle dispersion was assumed to be equal to the tracer 705 dispersion. The values of dispersion coefficient are 706 given in Table 1. Comparing the $\varepsilon_{D}$ values in Fig. 10 707 and those in Table 1, one could conclude that there 708 should be no significant difference between the pro709 posed and HLL models for low values of dispersion in 710 the laboratory tests.

711 Matching the laboratory data in limited cores by the 712 analytical model for flow in a semi-infinite reservoir 713 was suggested by Unice and Logan (2000). Fig. 11a 714 and b depict breakthrough curves calculated by the 715 analytical model (Eq. (C-2)) using the values of $\Lambda$ 716 and $\varepsilon_{D}$ from Tables 1 and 2.

717 From Fig. 11a and b it is apparent that both models 718 describe the experimental data equally well.

719 The difference between the filtration coefficients as 720 predicted by the different models (second and third 721 columns of the table) is not very high due to low 722 dispersion of the porous media used in laboratory 723 tests. A typical value of the filtration coefficient in 724 Table 2 is $\Lambda=1.4$, and hence the two models would

\section{t1.1 Table 1}

Summary of experiments by Ginn (2002) and Jin et al. (1997)

Test no Column Flow Dispersion Dispersivity Dimensionless

length $L$ rate $J_{\mathrm{w}}$ coeff. $D \quad \alpha_{D}(\mathrm{~cm}) \quad$ dispersion $\varepsilon_{D}$
(cm) $\quad(\mathrm{cm} / \mathrm{h}) \quad\left(\mathrm{cm}^{2} / \mathrm{h}\right)$

\begin{tabular}{lccccl}
\hline Exp.1 & 10.0 & 29.6 & 7.70 & 0.26 & 0.026 \\
Exp.2 & 10.0 & 2.96 & 0.53 & 0.18 & 0.018 \\
Exp.3 & 20.0 & 3.35 & 1.23 & 0.37 & 0.02 \\
Exp.4 & 20.0 & 3.19 & 1.13 & 0.35 & 0.02 \\
Exp.5 & 20.0 & 3.11 & 1.13 & 0.36 & 0.02 \\
Exp.6 & 10.5 & 2.99 & 0.76 & 0.25 & 0.02 \\
\hline
\end{tabular}
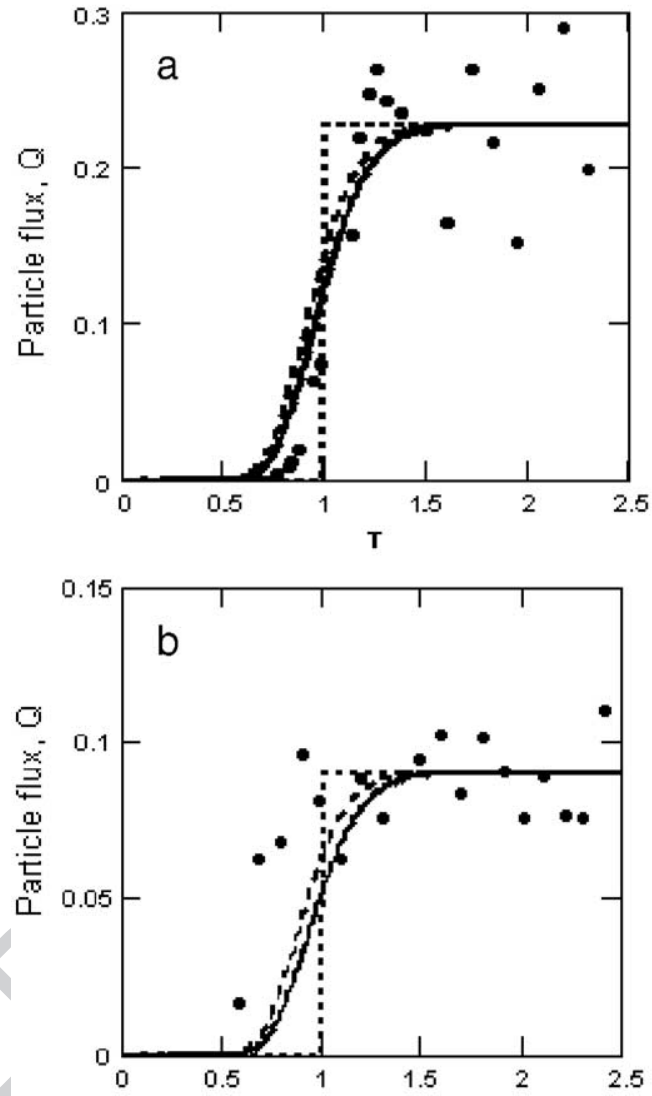

Fig. 11. Matching the breakthrough curves by the proposed and the HLL models (solid and dashed lines, respectively).

give different results for $\varepsilon_{D}$ greater higher 0.15 . The Table 1 shows that typical values of $\varepsilon_{D}$ for tests $3-6$ are 0.02 , and hence a noticeable difference between the two models cannot be anticipated.

The proposed model assumes that the capture rate is proportional to the total flux, while the HLL model assumes that the capture rate is proportional to the advective flux only. Consequently, the flux in the capture kinetics of the HLL model is lower than that of the presented model. Therefore, the filtration coefficient should be higher in the HLL model rather than in the proposed model in order to fit the same retaining kinetics value.

The comparison between the second and the third columns of Table 1 shows that the filtration coefficient predicted by the HLL model is higher than that predicted by the proposed model which confirms the above presented speculations.

However, due to low dispersion in laboratory tests, the difference in the values of the filtration coefficient values from the two models is not sufficiently high for validation of the proposed and HLL models.
725 
t2.1 Table 2

Filtration coefficient as obtained from breakthrough curves by the $\mathrm{t} 2.2$

$\mathrm{t} 2.3$

$\mathrm{t} 2.4$

$\mathrm{t} 2.5$

$\mathrm{t} 2.6$

$\mathrm{t} 2.7$

$\mathrm{t} 2.8$

$\mathrm{t} 2.9$

\section{7}

Test no and the HLL models

modeland by
dispersion free model

\section{Exp. 1}

Exp. 2

Exp. 3

Exp.4

Exp.5

12 /exp. 6

\begin{tabular}{ll}
0.59 & 0.6 \\
2.40 & 2.51 \\
1.48 & 1.52 \\
1.56 & 1.60 \\
1.38 & 1.42 \\
0.75 & 0.76 \\
\hline
\end{tabular}

748

The proposed and dispersion-free models give the

749 same equation for the inverse problem (Eq. (28)).

750 For large $\varepsilon_{D}$, the values of $\Lambda$ calculated by the two

751 models would differ significantly. For example, for

$752 \varepsilon_{D}=1$ and asymptotic outlet concentration $C=0.06$,

753 the filtration coefficients predicted by the proposed

754 and the HLL models are 2.8 and 7.1, respectively. The

755 data from natural reservoir cores rather than that from

756 sand columns may be used for validation of the model.

\section{9. Travelling dispersion wave}

758 Let us find the travelling wave solution for system 759 (Eq. (20)) with dynamic blocking filtration coefficient 760 (Eq. (5)):

$C=C(w), S=S(w), w=X-u T$

762 where $u$ is the unknown wave speed.

763 The travelling wave solution of the deep bed filtra-

764 tion system (Eq. (20)) is described by non-linear dy-

765 namic system (Eq. (F-7)) in plane $(C, S)$. The phase

766 portrait is shown in Fig. 12. The analysis of the dy-

767 namic system is analogous to that performed by $\mathrm{D}$.

768 Logan (2001), for HLL model.

769 The system has two singular points. The point $(0,0)$

770 correspond to the initial conditions (Eq. (12)), i.e. to the

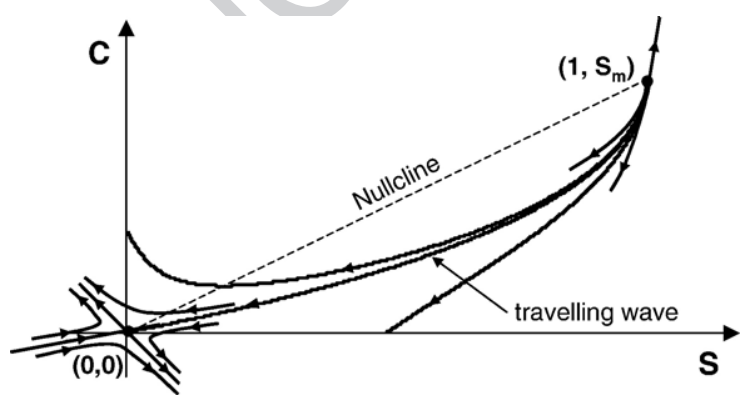

Fig. 12. Phase portrait of the dynamic system for dynamic blocking function. absence of particles before the injection, and point (1, $S_{\mathrm{m}}$ ), corresponds to the boundary condition (Eq. (14)), i.e. to the final equilibrium state $\left(1, S_{\mathrm{m}}\right)$, where $S_{\mathrm{m}}$ is the maximum number of retained particles per unit of rock volume. Point $(0,0)$ is a saddle point, the two orbits leaving the origin are unstable manifolds, and the two orbits entering the origin are stable manifolds. Point (1, $\left.S_{\mathrm{m}}\right)$ is an unstable repulsive node.

As shown in Fig. 12, there is only one trajectory that links the two singular points, and this trajectory is the travelling wave solution. The travelling wave joins initial and final equilibrium states of a system.

The travelling wave speed (Eq. (F-6)) was calculated in Appendix F:

$0<u=\frac{1}{1+S_{m}}<1$

At large length scale exceeding the travelling wave thickness, the wave (Eq. (29)) degenerates into shock wave. The speed (Eq. (30)) fulfils the Hugoniot condition of mass balance on the shock that corresponds to conservation law (Eq. (1)) (Bedrikovetsky, 1993). Therefore, the speed (Eq. (30)) for the proposed system (Eq. (20)) is the same as that for HLL model (Logan, 2001), since conservation law (Eq. (1)) is the same for either model.

The solution of initial-boundary value problem (Eqs. (12) and (14)) asymptotically tends to travelling wave for the case of blocking filtration function, (Eq. (5)). The travelling wave solution is invariant with respect to a shift along the axes $x$. The shift can be fixed at any time in order to provide an approximate solution for the initial-boundary value problem (Tikhonov and Samars-

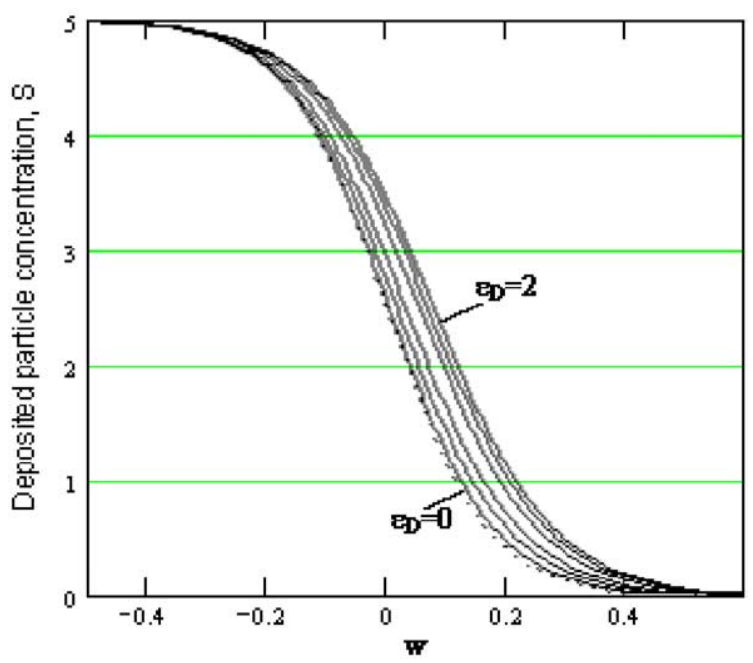

Fig. 13. Travelling wave solution without dispersion (traced line) and with dispersion (solid lines), for ${ }_{D}=0.03,0.1,0.2,0.5,1$ and 2 .
771

772

773

774

775

776

777

778

779

780

781

782

783

784

786

788

789

790

791

792

793

794

795

796

797

798

799

800

801

802 
803 kii, 1990). Calculations in Appendix F show that the 804 travelling wave fulfils the total mass balance for sus805 pended and retained particles (Eq. (1)) if and only if it 806 obeys the Goursat condition at the inlet $x=0$. It allows 807 choosing the shift at any time $T$ that the total mass 808 balance is fulfilled, see Eqs. (F-14) and (15).

809 The retained concentration profiles are shown in Fig. 81013 for several dispersion coefficients. The following 811 data were used: linear blocking function (Eq. (6)) $812 \Lambda(S)=10-2 S, c_{0}=100 \mathrm{ppm}$ and $\phi=0.2$. The disper813 sive wave $\left(\varepsilon_{D}>0\right)$ travels ahead of the dispersion-free 814 wave, and the wave velocities are equal. The higher is 815 the dispersion coefficient the more advanced is the 816 travelling wave.

\section{10. Three dimensional deep bed filtration with 818 dispersion}

819 Let us derive three dimensional deep bed filtration of 820 multi component suspension in porous media with size 821 exclusion mechanism of particle capture on the macro 822 scale. Particle populations with densities $\rho_{i}, i=1,2 . . n$, 823 flow in porous rock with velocities $U_{i}$.

824 Particle capture in one dimension is modelled in 825 Section 2 by a sieve sequence. The filtration coefficient $826 \lambda_{i}$ for each population is defined as a fraction of parti827 cles captured per unit of the particle trajectory. We 828 introduce the reference distance $l$ between the sieve 829 surfaces. Generally speaking, $l$ is a continuous function 830 of $(x, y, z)$, where $(x, y, z)$ is a point of three dimen831 sional flow domain. A sieve captures $\lambda_{i} l$-th fraction of 832 passing particles of $i$-th population, i.e. if $\rho_{i} U_{i}$ is a flux 833 of $i$-th population particles entering the "core" which is 834 perpendicular to the sieves, the particle capture rate is $835 \lambda_{i} l \rho_{i} U_{i}$, (Fig. 1).

836 The sieve surface has locally a plane form, so the 837 sieves filling the three dimensional domain form two838 dimensional vector bundle. Existence of a reference 839 distance $l$ between the sieve surfaces is consistent 840 with the assumption of integrability of the vector bun841 dle. Therefore, we consider the foliation case where the 842 sieves are located on the surfaces where a smooth 843 function $f(x, y, z)$ is constant.

844 For $i$-th population flux, the particle capture rate in a 845 reference volume $V$ is proportional to the flux projec846 tion on the vector perpendicular to the sieve. So, one847 dimensional product $\lambda_{i} l \rho_{i} U_{i}$ (Eq. (4)) is substituted by 848 the scalar product of the flux vector and the unit length 849 vector perpendicular to the sieve:

${ }_{850} \lambda_{i}\left\langle\frac{\nabla f}{|\nabla f|}, \rho_{i} U_{i}\right\rangle V$
Therefore, the particle mass balance for $i$-th population with the consumption rate (Eq. (31)) becomes:

$\frac{\partial \rho_{i}}{\partial t}+\operatorname{div}\left(\rho_{i} U_{i}\right)=-\lambda_{i}\left\langle\frac{\nabla f}{|\nabla \mathrm{f}|}, \rho_{i} U_{i}\right\rangle$

Introduce average mass density and velocity of the overall multi component flux

$\rho=\sum_{i} \rho_{i}, U=\sum_{i} \frac{\rho_{i} U_{i}}{\rho}$

The diffusive flux of $i$-th component around the front moving with the average velocity $U$ is defined as a difference between the $i$-th component flux moving with the $i$-th component velocity and that with the average velocity (Landau and Lifshitz, 1987; Nikolaevskij, 1990)

$\rho_{i} U_{i}=c_{i} \rho U-D_{i} \rho \nabla c_{i}$

Assuming incompressibility of the mixture

$\rho=$ const, $\operatorname{div} U=0$

and substituting Eqs. (34) and (35) into Eq. (32), we obtain the following form of the particle mass balance for $i$-th population accounting for particle dispersion and capture

$\frac{\partial c_{i}}{\partial t}+\left\langle U, \nabla c_{i}\right\rangle=D_{i} \Delta c_{i}-\lambda_{i}\left\langle\frac{\nabla f}{|\nabla f|}, c_{i} U-D_{i} \nabla c_{i}\right\rangle$

Opening brackets of the scalar product in right hand side of Eq. (36) and grouping terms in the left and right hand sides, we obtain

$$
\frac{\partial c_{i}}{\partial t}+\left\langle U-\lambda_{i} D_{i} \frac{\nabla f}{|\nabla f|}, \nabla c_{i}\right\rangle=D_{i} \Delta c_{i}-\lambda_{i}\left\langle\frac{\nabla f}{|\nabla f|}, U\right\rangle c_{i}
$$

Eq. (37) is a three dimensional generalization of Eq. (16). It allows describing the anisotropy capture effect where the filtration coefficient depends on the flow direction, while three dimensional generalization of HLL Eq. (18) can describe just a scalar (isotropic) particle capture.

The first term in the scalar product in the left hand side of Eq. (37) consists of the average flow velocity $U$ and the velocity with module $\lambda_{i} D_{i}$ directed perpendicular to sieve surfaces. So, the collective effect of dispersion with capture results in slowing down the advective particle flux.
868

856

858

860

862

863

864

865

866

870

872

873

874

876

878

879

880

883

884

885

886

887

888

889

890

891

892

893 


\section{11. Summary and conclusions}

895 The particle size exclusion capture rate in deep bed 896 filtration is proportional to the total particle flux includ897 ing both the advective and the dispersive flux compo898 nents. Therefore, the dispersion term must be present 899 not only in the particle balance equation but also in the 900 capture kinetics equation.

901 The outlet concentration for steady-state flow in a 902 limited core is completely determined by the particle 903 capture probability; therefore, it is independent of the 904 dispersion coefficient. The outlet concentration by the 905 model proposed is independent of dispersion, while 906 that by the traditional HLL model is dispersion 907 dependent.

908 The steady state flux profile in semi-infinite and 909 limited size porous media should be also dispersion910 independent, as the proposed model shows. The HLL 911 model exhibits dependency of steady state flux profile 912 on dispersion.

913 It allows concluding that for steady state flows the 914 proposed model exhibits physically coherent results, 915 while the traditional model exhibits physically unreal916 istic behaviour.

917 The collective effect of dispersion and capture on 918 deep bed filtration in the model proposed is a delay 919 in the propagation of the advective concentration 920 wave.

921 A constant filtration coefficient can be determined 922 from the asymptotical steady-state outlet concentration 923 during a transient coreflood test using the proposed 924 model without knowing the dispersion coefficient, 925 while the dispersion coefficient should be known in 926 order to calculate the filtration coefficient by the HLL 927 model.

928 The constant filtration coefficient as determined 929 from the asymptotical value of effluent concentration 930 using the proposed model is equal to that determined by 931 the dispersion-free model. Therefore, the HLL and the 932 proposed models show equally satisfactory fit with the 933 data of available experiments under small dispersivity. 934 Laboratory experiments in heterogeneous cores with 935 high dispersivity should be carried out in order to 936 validate the proposed model.

937 The travelling wave regime of deep bed filtration 938 with dispersion exists for the blocking type of filtra939 tion coefficient only. The velocity of the travelling 940 wave is determined by the maximum concentration 941 of retained particles and is independent of dispersion 942 coefficient. The higher is the maximum retained par943 ticle concentration the lower is the travelling wave 944 speed.
The proposed three dimensional model allows describing anisotropic particle capture while 3D HLL model describes only scalar (isotropic) capture of suspended particles.

\section{Uncited reference}

Harter et al., 2000

950

951

\section{Nomenclature}

c Suspended particles concentration

$c^{0} \quad$ Inlet suspended particles concentration

C Dimensionless suspended particle concentration

$D \quad$ Dispersion coefficient

$k^{0} \quad$ Original permeability

$l \quad$ Distance between sieves

$p \quad$ Pressure

$P \quad$ Dimensionless pressure

$q \quad$ Particle flux

$Q \quad$ Dimensionless particle flux

$S \quad$ Laplace coordinate

$S$ Dimensioless retained particles concentration Time

$t$

$T \quad$ Dimensionless time

$U$ Darcy's velocity

$v$ Delay term in the advective velocity

$x \quad$ Linear co-ordinate

$X \quad$ Dimensionless co-ordinate

$w \quad$ Transformation variable

$\alpha_{D} \quad$ Dispersivity

$\beta \quad$ Formation damage coefficient

$\varepsilon_{D} \quad$ Dimensionless dispersion coefficient

$\phi \quad$ porosity

$\lambda \quad$ Filtration coefficient

$\Lambda$ Dimensionless filtration coefficient

$\mu \quad$ Suspension viscosity

$\sigma \quad$ Retained particle concentration

952

953

954

955

956

957

958

959

960

961

962

963

964

965

966

967

968

969

970

971

972

973

974

975

976

977

978

979

980

\section{Acknowledgements}

981

The authors are grateful to Profs. Yannis Yortsos (South California University, USA), Oleg Dinariev (Institute of Earth Physics, Russian Academy of Sciences), Dan Marchesin (Institute of Pure and Applied Mathematics, Brazil) and Mikhael Panfilov (ENSG - INPL, Nancy, France) for the fruitful discussions. The collaboration with Adriano Santos (North Fluminense State University, UENF/Lenep, Brazil) is highly acknowledged.

Special thanks are due to Prof. Themis Carageorgos (UENF/Lenep) for support and encouragement.
982

983

984

985

986

987

988

989

990

991

992 


\section{Appendix A. Dimensionless governing equations}

994 Introduction of dimensionless variables and 995 parameters

$X=\frac{x}{L}, T=\frac{U t}{\phi L}, C=\frac{c}{c^{0}}, S=\frac{\sigma}{c^{0} \phi}, \Lambda(S)=\lambda(\sigma) L$,

$P=\frac{k_{0} p}{U \mu L}, Q=\frac{q}{c^{0} U}, \varepsilon_{D}=\frac{\alpha_{D}}{L}$

996 transforms the governing Eqs. (1) (2) (4) and (9) 998 to the following form:

$\frac{\partial}{\partial T}(C+S)+\frac{\partial Q}{\partial X}=0$

$999 \frac{\partial S}{\partial T}=\Lambda Q$

1000

$Q=C-\varepsilon_{D} \frac{\partial C}{\partial X}$

$-\frac{1}{\left(1+\beta \phi c^{0} S\right)} \frac{\partial P}{\partial X}=1$

1001

1002

1005

1006

The boundary conditions (Eqs. (13) and (15)) in dimensionless variables (Eq. (A-1)) take the form:

$X=0: Q=C-\varepsilon_{D} \frac{\partial C}{\partial X}=1$

$(\mathrm{A}-6)$

$X=1: \frac{\partial C}{\partial X}=0$

$(\mathrm{A}-7)$

1008 The simplified boundary condition (Eq. (14)) becomes:

$X=0: C=1$

101凹 The initial conditions (Eq. (12)) remain the same. 1013 Substituting the capture rate expression (Eq. (A-3)) 1014 into the mass balance Eq. (A-2), we obtain:

$\frac{\partial C}{\partial T}+\frac{\partial Q}{\partial X}=-\Lambda Q$

$(\mathrm{A}-9)$

101Б Substituting Eq. (A-4) into Eq. (A-9) yields the follow1018 ing parabolic equation:

1019

$\frac{\partial C}{\partial T}+v \frac{\partial C}{\partial X}=\varepsilon_{D} \frac{\partial^{2} C}{\partial X^{2}}-\Lambda C$

$(\mathrm{A}-10)$

$v=1-\Lambda \varepsilon_{D}$

$(\mathrm{A}-11)$

102@ Introduction of other dimensionless time, linear co1023 ordinate, pressure and filtration coefficient

$X^{\prime}=\lambda_{0} x, T^{\prime}=\frac{U \lambda_{0} t}{\phi}, \Lambda^{\prime}(S)=\frac{\lambda(\sigma)}{\lambda_{0}}, P^{\prime}=\frac{k_{0} p \lambda_{0}}{U \mu}$

$(\mathrm{A}-12)$ keeps Eqs. (A-2) (A-3) and (A-5) the same; Eq. (A-4) becomes

1024

1026

$Q=C-\varepsilon \frac{\partial C}{\partial X^{\prime}}, \varepsilon=\alpha_{D} \lambda_{0}$

\section{Appendix B. Flow in an infinite reservoir}

1028

1029

Let us consider flow in an infinite reservoir where, initially, water with particles was filling the semi-infinite reservoir $X<0$, and clean water was filling the semiinfinite reservoir $X>0$ (so-called Riemann problem):

$T=0: C(X, 0)=\left\{\begin{array}{l}1, X<0 \\ 0, X>0\end{array}\right.$

$(\mathrm{B}-1)$

Boundary conditions $C=0$ and $C=1$ must be satisfied at $X \rightarrow \infty$ and $X \rightarrow-\infty$, respectively.

The filtration coefficient is supposed to be constant.

The solution for deep bed filtration in an infinite reservoir Eqs. (A-10) and (B-1) can be obtained in explicit form (Polyanin, 2002):

1030

1031

1032

1033

$C(X, T)=\frac{1}{2}\left[\exp (-\Lambda T) \operatorname{erfc}\left(\frac{X-v T}{2 \sqrt{\varepsilon_{D} T}}\right)\right] \quad(\mathrm{B}-2)$

Appendix C. Transient solution for a semi-infinite reservoir

1042

1044

1045

Let us discuss the particulate suspension injection into a semi-infinite reservoir, $X>0$. The initial and boundary conditions are defined by Eqs. (12) (A-6) (A-7), respectively. The condition $C=0$ for a semiinfinite reservoir should be satisfied at $X \rightarrow \infty$.

The explicit solution of the problem is obtained by substitution

1046

1047

1048

1049

1050

1051

1052

$C(X, T)=\exp (-\Lambda T) w(X, T)$

and by Laplace transform in $T$ (Polyanin, 2002):

1053

$C(X, T)=\frac{1}{A} \exp \left[\frac{(v-A) X}{2 \varepsilon_{D}}\right] \operatorname{erfc}\left(\frac{X-A T}{B}\right)$

$-\frac{1}{A} \exp \left[\frac{(v+A) X}{2 \varepsilon_{D}}\right] \operatorname{erfc}\left(\frac{X+A T}{B}\right)$

$-\frac{(2-v)}{2 \varepsilon_{D}} \exp \left(\frac{X}{\varepsilon_{D}}\right)$

$\times \int_{0}^{T} \operatorname{erfc}\left(\frac{X+(2-v) t}{2 \sqrt{\varepsilon_{D} t}}\right) d t$

$(\mathrm{C}-2)$

$A=\sqrt{v^{2}+4 \Lambda \varepsilon_{D}}$

$(\mathrm{C}-3)$

$B=2 \sqrt{\varepsilon_{D} T}$

$(\mathrm{C}-4)$ 
1058 The solution (Eq. (C-2)) reaches steady state as $T \rightarrow \infty$ :

$$
C(X, \infty)=\frac{1}{1+\Lambda \varepsilon_{D}} \exp (-\Lambda X)
$$

$106 \oplus$ Formula Eq. (C-5) is a steady-state solution of the

1062

1063

1064

$$
Q(X)=\exp (-\Lambda X)
$$

1066

1067

1068

1069

1070

1071

1072

1073

1074

1075

1076

1078

1079

1080

1081

1083

1084

1085

1086

\section{Appendix D. Filtration in semi-infinite reservoir with simplified inlet boundary conditions}

Let us discuss the simplified case where dispersion is neglected in the inlet boundary conditions. Eq. (A10 ) is subject to initial condition Eq. (12), inlet boundary condition (Eq. (A-8)/Eq. (14)); the condition $C=0$ must be satisfied as $X \rightarrow \infty$.

The problem is solved using the Laplace transform in $T$ (Polyanin, 2002):

$$
\begin{aligned}
C(X, T)= & \frac{1}{2}\left[\exp \left(\frac{X}{\varepsilon_{D}}\right) \operatorname{erfc}\left(\frac{X+M T}{B}\right)\right. \\
& \left.+\exp (-\Lambda X) \operatorname{erfc}\left(\frac{X-M T}{B}\right)\right]
\end{aligned}
$$

$M=1+\Lambda \varepsilon_{D}$

where constant $B$ is given by formula Eq. (C-16).

The solution (Eq. (D-1)) tends to steady-state asymptotic as $T \rightarrow \infty$ :

$C(X, \infty)=\exp (-\Lambda X)$

\section{Appendix E. Steady-state solution for filtration in finite cores}

The equation for steady state in finite cores corresponds to zero time derivative in Eq. (A-9):

$\frac{d Q}{d X}=-\Lambda Q$

The direct integration of the ordinary differential Eq. (E-1) taking into account the inlet boundary condition (Eq. (A-6)) results in the expression for the particle flux profile

$Q=\exp (-\Lambda X)$

$(\mathrm{E}-2)$

which coincides with the flux profile (Eq. (C-18)) in semi-infinite media.

Substitution of the flux expression Eq. (E-2) into Eq. (A-4) leads to a first-order ordinary differential equa- tion for suspended concentration profile. The solution

1098 that takes account of the outlet boundary condition Eq. (A-7) is given by

1099

1100

$$
\begin{aligned}
C(X)= & \frac{1}{1+\Lambda \varepsilon_{D}}[\exp (-\Lambda X) \\
& \left.+\Lambda \varepsilon_{D} \exp \left(\frac{1}{\varepsilon_{D}}(X-1)-\Lambda\right)\right] .
\end{aligned}
$$

The inlet concentration is calculated from Eq. (E-3):

$110 B$

$$
C(0)=\frac{1}{1+\Lambda \varepsilon_{D}}\left[1+\Lambda \varepsilon_{D} \exp \left(-\frac{1}{\varepsilon_{D}}-\Lambda\right)\right]
$$

The outlet concentration at $X=1$ is also obtained directly from Eq. (E-3):

1106

1107

$C(1)=\exp (-\Lambda)$

The outlet boundary condition (A-7) implies that the particle flux and the suspended concentration coincide at $X=1$.

The retention dynamics can be found from Eq. (A-3) using the expression for the particle flux (Eq. (E-2)):

$S(X, T)=\Lambda T \exp (-\Lambda X)$

\section{Appendix F. Travelling wave solutions}

Let us find travelling wave solutions

$C=C(w), S=S(w), w=X-u T$

for system Eq. (20), where $u$ is an unknown wave speed.

The corresponding system of ordinary differential equations as obtained from Eq. (20) is

$\frac{d S}{d w}=-\Lambda(S)(C+S)$

$\frac{d C}{d w}=\frac{1}{\varepsilon_{D}}[(1-u) C-u S]$

The initial condition (12) for system Eq. (20) was already used during integration (Eqs. (21)-(24)), so the dynamic system (Eqs. (F-2) and (F-3)) fulfils the corresponding boundary condition:

$w \rightarrow+\infty \quad: C \rightarrow 0 ; S \rightarrow 0$

The existence of the limited solution at minus infinity implies for Eq. (F-2) that $\Lambda\left(S_{\mathrm{m}}\right)=0$, i.e. the filtration coefficient should be a blocking function, see Eq. (5). The corresponding boundary condition at minus infinity for the dynamic system (Eqs. (F-2) and (F-3)) is
1116

1117

1118

1100

1111

1112

1113

1114

$112 \theta$

1121

1122

1123

1124

$112 \overline{6}$

1128

1129

1130

1133

1134

1135

1136

1137 
1138 obtained from the boundary condition (14):

$w \rightarrow-\infty: C \rightarrow 1 ; S \rightarrow S_{m}$

1140

1142

$0<u=\frac{1}{1+S_{\mathrm{m}}}<1$

1143

1146

1147

1148

1149

1150

$\frac{d C}{d S}=-\frac{(1-u) C-u S}{\varepsilon_{D} \Lambda(S)(C+S)}$

$115 B$

1154

1155

1156

1157

1158

1159

1160

1161

1162

$w(S)=-\int^{S} \frac{1}{\Lambda(s)(C(s)+s)} d s+$ const. $\quad(\mathrm{F}-8)$

1163 The solution of the initial-boundary value problem 1166 (Eqs. (12) and (14)) tends asymptotically to the travel1167 ling wave (Eqs. (F-7) and (F-8)). It happens when $T$ 1168 tends to infinity along each characteristic $1169 \quad X-u T=w=$ const:

$\left.\lim _{T \rightarrow \infty} C(X, T)\right|_{w=\text { const }}=\lim _{T \rightarrow \infty} C(w+u T, T)=C(w)$

$\left.\lim _{T \rightarrow \infty} S(X, T)\right|_{w=\text { const }}=\lim _{T \rightarrow \infty} S(w+u T, T)=S(w)$

$(\mathrm{F}-10)$ kinetics (Eq. (A-3)) and dividing variables in the ordinary differential equation, we obtain

1180

1181

$X=0: T=\int_{0}^{S(0, T)} \frac{d y}{\Lambda(y)}=\Phi(S)$

The expression for retained concentration is obtained from Eq. (F-11) applying the inverse function

1182

1185

$S(0, T)=\Phi^{-1}(T)$

$(\mathrm{F}-12)$

The travelling wave solution is invariant with respect to a shift $(X, T) \rightarrow(X+$ const, $T)$. Let us fix the constant $w$ in Eq. (F-8) for each moment $T$ in such a way, that the inlet retained concentration is the same as that in the solution of the initial-boundary value problem (Eq. (F-12)). So, Eq. (F-8) takes the form:

$$
w(S, T)=-\int_{\Phi^{-1}(T)}^{S} \frac{1}{\Lambda(s)(C(s)+s)} d s-u T
$$

Finally, the delay term in the travelling wave variable is chosen for any $T$ in such a way, that the Goursat condition (Eq. (F-12)) is fulfilled.

Let us show that it provides with the total mass balance for the conservation law (Eq. (1)).

Substituting the travelling wave form (Eq. (F-1)) into the mass balance (Eq. (A-2))

$$
\int_{-u T}^{\infty}(C+S) d w=T
$$

and performing integration in $x$, from the Eq. (F-13) we obtain

1203 1205

$$
\begin{aligned}
T & =\int_{-u T}^{\infty}(C+S) d w=\int_{0}^{S(-u T)} \frac{d s}{\Lambda(s)} \\
& =\Phi(S(-u T))=\Phi(S(0, T))
\end{aligned}
$$

So, the solution (Eq. (F-13)) fulfils the integral mass balance for the domain $0<X<8$.

Solution in the plane $(X, T)$ for the retained particle concentration can be obtained by substituting $w=X-u T$ into Eq. (F-13):

1196

1197

1198

1199

1200

1201

1202

$X(S, T)=-\int_{\Phi^{-1}(T)}^{S} \frac{1}{\Lambda(s)(C(s)+s)} d s \quad(\mathrm{~F}-16)$

Eq. (F-16) is an approximate solution for initialboundary value problem (Eqs. (12) and (14)).
1208

1209

1210

1211

1212

1213

1216 


\section{References}

\section{8}

1219 Bedrikovetsky, P.G., 1993. Mathematical Theory of Oil and Gas 1220 Recovery. Kluwer Academic Publishers, Dordrecht.

1221 Bedrikovetsky, P.G., Marchesin, D., Checaira, F., Serra, A.L., 1222 Resende, E., 2001. Characterization of deep bed filtration system 1223 from laboratory pressure drop measurements. J. Pet. Sci. Eng. 64, 1224167.

1225 Bedrikovetsky, P., Marchesin, D., Hime, G., Alvarez, A., Marchesin, 1226 A.O., Siqueira, A.G., Souza, A.L.S., Shecaira, F.S., Rodrigues, 1227 J.R., 2002. "Porous media deposition damage from injection of 1228 water with particles," VIII Ecmor European Conference on Math1229 ematics in Oil Recovery, Sept. 3-6, Austria, Leoben.

1230 Bolster, C.H., et al., 1998. A method for calculating bacterial depo1231 sition coefficients using the fraction of bacteria recovered from 1232 laboratory columns. Environ. Sci. Technol. 32, 1329.

1233 Danckwerts, P.V., 1953. Continuous flow systems: distribution of 1234 residence times. Chem. Eng. Sci. 2, 1.

1235 Elimelech, M., et al., 1995. Particle Deposition and Aggregation: 1236 Measurement, Modelling, and Simulation. Butterworth-Heine1237 mann, Oxford, England.

1238 Ginn, T.R., 2002. A travel approach to exclusion on transport in 1239 porous media. Water Resour. Res. 38, 1129.

1240 Grolimund, D., et al., 1998. Transport of in situ solubilized colloidal 1241 particles in packed soil columns. Environ. Sci. Technol. 32, 3562. 1242 Harter, T., et al., 2000. Colloid transport and filtration of Cryptospo1243 ridium parvum in sandy soils and aquifer sediments. Environ. Sci. 1244 Technol. 34, 62.

1245 Herzig, J.P., Leclerc, D.M., Goff, P.L., 1970, May. Flow of suspen1246 sions through porous media - application to deep filtration. Ind. 1247 Eng. Chem. 62 (5), 8.

1248 Iwasaki, T., 1937. Some notes on sand filtration. J. Am. Water Works 1249 Assoc. 29, 1591.

1250 Jensen, J.L., et al., 1997. J. Statistics for Petroleum Engineers and 1251 Geoscientists. Prentice Hall PTR, New Jersey.

1252 Jin, Y., et al., 1997. Sorption of viruses during flow through saturated 1253 sand columns. Environ. Sci. Technol. 31, 548.

1254 Kampen, Van N.G., 1984. Stochastic Processes in Physics and Chem1255 istry. North-Holland, Amsterdan-Oxford.

1256 Kechagia, P., Tsimpanogiannis, I., Yortsos, Y., et al., 2002. On the 1257 upscaling of reaction-transport processes in porous media with 1258 fast or finite kinetics. Chem. Eng. Sci. 57 (13), 2565.

1259 Khilar, K.C., Fogler, H.S., 1998. Migration of Fines in Porous Media. 1260 Kluwer Academic Publishers, Dordrecht-Boston-London.

1261 Kretzschmar, R., Barmettler, K., et al., 1997. Experimental determi1262 nation of colloid deposition rates and collision efficiencies in 1263 natural porous media. Water Resour. Res. 33, 1129.

1264 Kuhnen, F., Barmettler, K., et al., 2000. Transport of iron oxide 1265 colloids in packed quartz sand media. J. Colloid Interface Sci. 1266231

1315
Lake, L.W., 1989. Enhanced Oil Recovery. Prentice Hall, Englewood Cliffs.

Landau, L.D., Lifshitz, E.M., 1987. Fluid mechanics. (2nd ed.). Course of Theoretical Physics, vol. 6. Pergamon Press, Oxford.

Logan, D.J., 2001. Transport Modeling in Hydrogeochemical Systems. Springer.

Massei, N., et al., 2002. Transport of particulate material and dissolved tracer in a highly permeable porous medium: comparison of the transfer parameters. J. Contam. Hydrol. 57, 21.

Nikolaevskij, V.N., 1990. Mechanics of Porous and Fractured Media. World Scientific, New Jersey-Hong Kong.

Pang, S., Sharma, M.M., 1994. A model for predicting injectivity decline in water injection wells. SPE paper 28489 presented at 69th Annual Technical Conference and Exhibition held in New Orleans, LA, 25-28 September.

Payatakes, A.C., et al., 1974. Application of porous medium models to the study of deep bed filtration. Can. J. Chem. Eng. 52, 727.

Polyanin, A.D., 2002. Handbook on linear partial differential equations for scientists and engineers. Chapman and Hall CRC Press, Boca Raton-London.

Santos, A., Bedrikovetsky, P., 2005. Size exclusion during particle suspension transport in porous media: stochastic and averaged equations. J. Comput. Appl. Math 3.

Sharma, M.M., Yortsos, Y.C., 1987a. Transport of particulate suspensions in porous media: model formulation. AIChe J. 33 (10), 1636.

Sharma, M.M., Yortsos, Y.C., 1987b. A network model for deep bed filtration processes. AIChE J. 33 (10), 1644-1653.

Sharma, M.M., Yortsos, Y.C., 1987c. Fines migration in porous media. AIChE J. 33 (10), 1654-1662.

Sorbie, K., 1991. Polymer-improved Oil Recovery. CRC Press, Boca Raton-London.

Tiab, D., Donaldson, E.C., 1996. Petrophysics. Gulf Publishing, Houston.

Tikhonov, A.N., Samarskii, A.A., 1990. Equations of Mathematical Physics. Dover, New York.

Tufenkji, N., et al., 2003. Interpreting deposition patterns of microbial particles in laboratory-scale column experiments. Environ. Sci. Technol. 37, 616.

Unice, K.M., Logan, B.E., 2000. Insignificant role of hydrodynamic dispersion on bacterial transport. J. Environ. Eng. 126, 491.

van Genuchten, Th.W., 1981. Analytical solutions for chemical transport with simultaneous adsorption, zero-order production and first-order decay. J. Hydrol. 49, 213.

Wennberg, K.E., Sharma, M.M., 1997. Determination of the filtration coefficient and the transition time for water injection. Proceedings of the SPE European Formation Damage Conference, SPE 38181, The Hague, The Netherlands, June 2-3.
1267 